\title{
Anidulafungin in the treatment of invasive fungal infections
}

\author{
Kathryn Sabol \\ Tawanda Gumbo \\ University of Texas Southwestern \\ Medical Center, Dallas, TX, USA
}

Correspondence: Tawanda Gumbo

Division of Infectious Diseases,

UT Southwestern Medical Center,

5323 Harry Hines Blvd.,

Dallas, TX 75390-9113, USA

Tel + I $21464899 \mid 4$

Fax $+|2| 4648274 \mid$

Email tawanda.gumbo@utsouthwestern.edu

\begin{abstract}
More antifungal agents have reached clinical use in the past two decades than at any other time. The echinocandins have been a welcome addition to this group, with the latest being anidulafungin. There are several lines of evidence to support anidulafungin's role as primary therapy for the treatment of invasive candidiasis in non-neutropenic patients, and as alternative therapy to fluconazole in patients with esophageal candidiasis with azole intolerance or triazole-resistant Candida. Pharmacokinetic-pharmacodynamic studies in animals have demonstrated superior efficacy, defined as maximal microbial kill, when compared to fluconazole, regardless of the fluconazole susceptibility of the Candida species. These studies, as well as dose-effect studies in patients, also support the currently recommended dose of anidulafungin. A well designed randomized controlled trial has demonstrated anidulafungin's efficacy in patients with invasive candidiasis. In this paper, we argue that anidulafungin may be preferable to fluconazole for the treatment of candidemia. However, as of yet, the difference between anidulafungin and the other two licensed echinocandins as first-line therapy for invasive candidiasis is unclear. On the other hand, there is insufficient evidence as of yet to support first-line use of anidulafungin in patients with neutropenia or aspergillosis.
\end{abstract}

Keywords: anidulafungin, pharmacokinetics-pharmacodynamics, efficacy, candidiasis

\section{Introduction to management of serious invasive fungal infections}

Fungal infections are a growing global problem. The most common global mycoses are due to infections by Candida species, Cryptococcus neoformans, Aspergillus species, and other molds. The impact of this group of fungal infections has increased as the population of immunosuppressed patients has increased due to the AIDS pandemic, advances in immunosuppressive therapies, invasive instrumentation of hospitalized patients, and an aging global population. For decades, the basis of most antifungal therapy has been the polyenes, specifically amphotericin B-based medications, the cytosine analogue 5-fluorocytosine, and triazole compounds. Polyene-based therapy is plagued by the problem of toxicity. Azoles and 5-fluorocytosine have the limitation of resistance emergence in yeast infections, especially problematic in the therapy of Candida glabrata (Nguyen et al 1996; Gumbo et al 1999; Alexander et al 2005). Indeed, even after apparent therapeutic success with these agents, recurrence of infection has been noted months to years later (Nasser et al 1997; Clancy et al 2000; Gumbo et al 2002). Thus, development of new classes of antifungal agents was imperative. One class of new antifungal agents that have reached clinical use is that of echinocandins, of which anidulafungin is the latest member. The echinocandins have activity against Candida and Aspergillus species, but not $C$. neoformans.

\section{History of anidulafungin}

Anidulafungin is a semisynthetic product of echinocandin B, itself a fermentation product of the mold Aspergillus nidulans. It was developed by Eli Lily, underwent preclinical and clinical development at Vicuron Pharmaceuticals, and was sold to Pfizer where it is marketed under the name Eraxis ${ }^{\mathrm{TM}}$. It received approval from the Food and 
Drug Administration in February 2006. Studies for its use in candidemia are continuing under the sponsorship of Pfizer.

\section{Mode of action and spectrum of activity}

The polymers glucan, mannose, and chitin are responsible for fungal cell wall shape and strength. Glucan is made up of 3 helically entwined glucose polymers linked by $\beta-1,3-$, $\alpha-1,3$ or $\beta-1,6$-bonds. Anidulafungin inhibits enzyme complex $1,3-\beta$-D-glucan synthase and thereby inhibits fungal 1,3- $\beta$-D-glucan synthesis. This leads to lysis of the fungal cell wall, and cell death. It is not surprising that when resistance develops, whether in the laboratory or in patients with candidiasis, the isolates often contain a mutation in the gene that encodes for components of the 1,3- $\beta$-D-glucan synthase enzyme complex such as FKS1 and FKS-2 (Kurtz et al 1996). Mutations in the $f k s /$ gene lead to glucan synthase activity that is more resistant to the effect of echinocandins than the wild type enzyme. Fungi that have an abundance of $\beta$-1,3-bonds will, in general, be susceptible to anidulafungin, at least in the laboratory. The susceptibility of a variety of fungal pathogens to anidulafungin are shown in Table 1 (adapted and modified from Gumbo et al 2007a), which summarizes data from a variety of laboratory studies of clinical isolates (Espinel-Ingroff 1998; Oakley 1998; Espinel-Ingroff 2003; Serrano et al 2003). Fusarium species, Trichosporon

Table I Susceptibility of common fungal pathogens to anidulafungin

\begin{tabular}{lll}
\hline Species & $\begin{array}{l}\text { Number of } \\
\text { isolates tested }\end{array}$ & $\begin{array}{l}\text { Anidulafungin } \text { MEC }_{90} \\
\text { or } \mathbf{M I C}_{90} \text { (mg/L) }\end{array}$ \\
\hline Candida species & 733 & 0.03 \\
C. albicans & 458 & 0.13 \\
C. glabrata & 391 & 2 \\
C. parapsilosis & 307 & 0.13 \\
C. tropicalis & 50 & 0.13 \\
C. krusei & 20 & 0.25 \\
C. lusitaniae & 18 & 0.06 \\
C. dubliniensis & & \\
Cryptococcus & 10 & $>16$ \\
neoformans & & \\
Aspergillus species & 28 & $<0.03$ \\
A. fumigatus & 19 & $<0.03$ \\
A. flavus & 9 & $<0.03$ \\
A.niger & 8 & $<0.03$ \\
A. glaucus & 8 & 0.003 \\
A. terreus & - \\
Penicillium species & & \\
Pseudoallescheria & & 2.5 \\
boydii & 6 & $>16$ \\
Rhizopus arrhizus & 5 & \\
\hline
\end{tabular}

beigelii, Rhizopus species, and C. neoformans are resistant to anidulafungin. Anidulafungin has low MICs against mycelial forms of $H$. capsulatum, B. dermatitidis, and C. immitis, but high MICs against the yeast forms (Espinel-Ingroff 1998).

\section{Administration, distribution, and elimination of anidulafungin}

Anidulafungin is FDA approved for the treatment of invasive candidiasis, candidemia and esophageal candidiasis. Recommended doses and infusion volumes are shown in Table 2 (Pfizer Inc. 2006). Anidulafungin requires a companion diluent, $20 \%$ weight/weight dehydrated alcohol, for reconstitution. The solution can be further diluted in either 5\% dextrose or normal saline solutions and administered at an infusion rate not exceeding $1.1 \mathrm{mg} / \mathrm{min}$. The solution is compatible with most other intravenous solutions, with notable exceptions including ertapenem, sodium bicarbonate, and amphotericin B (Trissel and Ogundele 2005). No oral alternative is available due to poor oral bioavailability $(<5 \%$ ), thus all recommended dosing is intravenous. After systemic administration of anidulafungin, murine studies demonstrate that anidulafungin persists in tissues for many days after a single dose of anidulafungin (Gumbo et al 2006). The time to peak concentrations in tissue was markedly slower than time to peak concentrations in the serum. The concentrations that are achieved are proportional to dose, indicating linear pharmacokinetics. The volume of distribution in adult patients is $0.6 \mathrm{~L} / \mathrm{kg}$, close to the total body water volume (Dowell et al 2004)). Anidulafungin is eliminated by non-enzymatic degradation from a ring form into a linear peptide, which is then degraded to tertiary products by nonspecific peptidases in human plasma. The serum terminal half-life is estimated to be nearly 1 day. Elimination of parent compound and degradation products is via the fecal route, with $<1 \%$ excreted via the urine. Therefore, from a xenobiotic metabolism standpoint, one would not expect dose adjustments in patients with renal or hepatic impairment. This has been confirmed in clinical studies (Dowell et al 2003).

\section{Population pharmacokinetics anidulafungin}

Clinicians at a patient's bedside often need to adjust doses of antimicrobials to individualize antibiotic therapy, given the demographic and pathophysiological conditions that are particular to the patient. In order to achieve this successfully, population pharmacokinetic analysis studies need to be performed to account for patient-to-patient variability, and the reasons for such variability. Dowell and colleagues performed population pharmacokinetic analysis on steady state serum 
Table 2 Recommended adult doses and infusion volumes for anidulafungin

\begin{tabular}{lll}
\hline Indication & Dosing schedule & Infusion volume \\
\hline Invasive candidiasis and candidemia & $200 \mathrm{mg}$ intravenous loading dose, then & $500 \mathrm{~mL}$ in loading dose; $250 \mathrm{~mL}$ \\
& $100 \mathrm{mg}$ iv daily & in maintenance dose \\
Esophageal candidiasis & $100 \mathrm{mg}$ intravenous loading dose, then & $250 \mathrm{~mL}$ in loading dose; $100 \mathrm{~mL}$ \\
& $50 \mathrm{mg}$ iv daily & in maintenance dose \\
\hline
\end{tabular}

Based on package insert (Pfizer Inc. 2006).

concentrations of 129 patients who were being treated for a variety of fungal infections with anidulafungin daily infusions in one of three doses of $50 \mathrm{mg}, 75 \mathrm{mg}$, or $100 \mathrm{mg}$ (Dowell et al 2004). The clearance of anidulafungin was increased with increases in body weight, a diagnosis of invasive candidiasis, and being male. However, these particular demographic and pathological features could only explain $20 \%$ of the intersubject variability in clearance, and therefore are considered to be clinically insignificant. The implication is that there is no need for anidulafungin dose adjustments between patients, even when patients are of different weight and gender.

\section{Pharmacokinetic-pharmacodynamic studies}

In order to determine optimal doses and dosing schedules, pharmacokinetic- pharmacodynamic (PK-PD) are performed. In such studies, the drug exposures expressed as PK-PD indices such as the area under the concentration-time curve to $\mathrm{MIC}$ (AUC/MIC), peak concentration to $\mathrm{MIC}\left(\mathrm{C}_{\max } / \mathrm{MIC}\right.$ ) or the time that drug concentration is above the $\mathrm{MIC}\left(\mathrm{T}_{\mathrm{MIC}}\right)$, are related to antimicrobial effect. If a drug's microbial kill correlates best with AUC/MIC or $\mathrm{C}_{\max } / \mathrm{MIC}$ ratio then administration of the same cumulative dose as intermittent doses would not compromise efficacy. However, if the drug's efficacy is best associated with $\mathrm{T}_{\text {MIC }}$, then more frequent dosing such as several times a day is better, while more extended dosing intervals would reduce efficacy. Data on the particular PK-PD index that best explains anidulafungin effect have been somewhat ambiguous. In a rabbit model of disseminated candidiasis and pulmonary aspergillosis the PK-PD index associated with effect could not be clearly distinguished (Groll et al 2001). Drugs within the same pharmacological class often have effect linked to the same PK-PD index. When Andes et al and Wiederhold et al examined other echinocandin agents, namely caspofungin and HMR 3270, they found that the $\mathrm{C}_{\max } / \mathrm{MIC}$ was most closely linked to antifungal effect (Andes et al 2003; Wiederhold et al 2004). We too have performed PK-PD studies of the echinocandins caspofungin and micafungin (Louie et al 2005; Gumbo et al 2007b). We found that for this class of agents, it is more the tissue concentration at the site of effect that correlates with microbial kill than that which we measure in serum. This has important implications in those echinocandins such as anidulafungin and caspofungin which persist in the tissues for many days, as opposed to micafungin which does not. If the infected animals are sacrificed early or prior to the full effect of the drug being achieved, it would be difficult to identify the PK-PD index most closely associated with effect. Taking this into consideration, we found that AUC/MIC best correlated with echinocandin antifungal effect. Thus, once-a-day, or even a dosing schedule once every few days should be efficacious. More extended anidulafungin dosing intervals, though, have not yet been examined in clinical trials. However, every-otherday echinocandin dosing has already been demonstrated to be as efficacious as daily therapy for the sister echinocandin, micafungin (Buell et al 2005). In the meantime, we have performed studies to determine the AUC/MIC exposure associated with optimal anidulafungin effect in mice with persistent neutropenia related candidemia, and compared the efficacy of anidulafungin versus fluconazole (Gumbo et al 2006). The AUC/MIC associated with optimal microbial effect in the mice, in an inhibitory sigmoid $\mathrm{E}_{\max }$ analysis, was an exposure which is easily be achieved by a $100 \mathrm{mg}$ dose in humans given the Candida $\mathrm{MIC}_{90}$ in clinical isolates. It is interesting that in an inhibitory sigmoid $\mathrm{E}_{\max }$ model analysis performed by Krause et al one of the factors associated with optimal anidulafungin therapy in patients with esophageal candidiasis was an AUC $>35 \mathrm{mg}^{*} \mathrm{~h} / \mathrm{L}$ (Dowell et al 2003). This exposure is also easily surpassed by an intravenous anidulafungin dose of $100 \mathrm{mg}$ a day in adults, which achieves a 0-24 h AUC of 110 $\mathrm{mg}^{*} \mathrm{~h} / \mathrm{L}$ (Pfizer Inc. 2006). Importantly, in our mouse study, the maximal microbial kill $\left(\mathrm{E}_{\max }\right)$ of anidulafungin was superior to that of fluconazole, whether the Candida strain utilized was susceptible to fluconazole, was susceptible-dose dependent, or fluconazole resistant. Thus, in the neutropenic animal model anidulafungin was more efficacious than fluconazole.

\section{Efficacy of anidulafungin: evidence from clinical trials}

Anidulafungin has been compared with fluconazole for the treatment of esophageal candidiasis in a randomized, 
double-blind, double-dummy study (Krause et al 2004b). Three hundred patients with endoscopically proven esophageal candidiasis were randomized to the anidulafungin arm and 301 to the fluconazole arm. Patients received a $100 \mathrm{mg}$ intravenous loading dose of anidulafungin followed by $50 \mathrm{mg}$ iv daily, or a $200 \mathrm{mg}$ loading dose of oral fluconazole followed by $100 \mathrm{mg}$ daily for 14-21 days. Assessments such as endoscopic response as well as clinical and microbiologic response, were performed at baseline and at end of therapy, with the clinical and microbiologic responses also assessed at a follow-up visit 2 weeks after the completion of therapy. The most common Candida species isolated were C. albicans (91\%) and C. glabrata ( 2\%). An intent-to-treat analysis at the end of treatment revealed similar success rates between anidulafungin $(86.7 \%)$ and fluconazole (88\%). However, at the 2 -week follow up visit, nearly $36 \%$ of patients in the anidulafungin arm had relapsed compared to only $10.5 \%$ in the fluconazole group. This problem of higher relapse in patients treated with anidulafungin versus fluconazole is thrown into doubt by the fact that more patients in the fluconazole arm (58/300) were started on antiretroviral therapy (ART) during antifungal therapy compared to those in the anidulafungin arm (26/301) ( $p<0.001$ by our analysis). Thus, higher relapse rates in the anidulafungin arm could be explained by ART therapy, or lack thereof. On the other hand, high relapse rates have been seen in patients with Candida esophagitis treated with the other echinocandins such as caspofungin (Arathoon et al 2002) and micafungin (de Wet et al 2004), so that it is still plausible, even taking ART into consideration, that anidulafungin therapy could lead to higher relapse rates.

In a Phase II dose-ranging study the safety and efficacy of anidulafungin in the treatment of invasive candidiasis and/or candidemia was evaluated in 123 patients (Krause et al 2004a). Adult patients were randomized to receive daily intravenous anidulafungin of $50 \mathrm{mg}, 75 \mathrm{mg}$ or $100 \mathrm{mg}$ for up to 14 days after resolution of infection, following a one-time loading dose (double the daily dose) on the first day. The most common isolates were C. albicans (53\%), C. glabrata (31\%), and C. parapsilosis (9\%). Global response (clinical and microbiological) rates at the end of treatment were $84 \%$ in the $50 \mathrm{mg}$ group, $90 \%$ in the $75 \mathrm{mg}$ group and $89 \%$ in the $100 \mathrm{mg}$ group. These results suggest that the dose of $100 \mathrm{mg}$ a day is on the optimal effect portion of the dose-response curve. This is also consistent with animal PK-PD studies. Taken together, this indicates that a daily dose of $100 \mathrm{mg}$ would be the optimum dose to study in randomized controlled studies versus standard therapy.

Fluconazole has been the standard therapy for invasive candidiasis in non-neutropenic patients since the early 1990s, due to both good efficacy and safety (Rex et al 1994). Amphotericin $\mathrm{B}$ is equally efficacious, but is limited by toxicity. The efficacy of fluconazole was recently compared with that of anidulafungin in the treatment of invasive candidiasis (Reboli et al 2007). In this double-blind study, 261 patients were enrolled and then stratified by APACHE score and presence of neutropenia. Patients were randomized to treatment with anidulafungin (200 mg loading dose then $100 \mathrm{mg}$ daily) or intravenous fluconazole ( $800 \mathrm{mg}$ loading dose then $400 \mathrm{mg}$ daily), with the option to switch to oral fluconazole therapy after at least 10 days of intravenous therapy. Therapy was continued for 14 days after a negative blood culture and absence of signs and symptoms. The most common isolates were C. albicans (61.6\%), C. glabrata (20.4\%), and C. parapsilosis (11.8\%). Successful outcomes were achieved in $75.6 \%$ treated with anidulafungin compared to $60.2 \%$ treated with fluconazole group (15.4\% difference, 95\% confidence interval: 3.9-27.0). Thus anidulafungin was superior to standard therapy. Patients receiving anidulafungin had higher rates of successful response for all Candida isolates with the exception of $C$. parapsilosis. However, the superiority of anidulafungin may be questioned due to a potential center effect. One center enrolled a total of 25 patients, and 14 of 15 patients treated with anidulafungin $(93.3 \%)$ at the center compared to 5 of 10 patients treated with fluconazole $(50 \%)$ had successful response. Removal of patients from this center makes anidulafungin non-inferior to fluconazole and not superior.

\section{Safety, tolerability, common adverse events}

Krause et al have reported an overall adverse event rate of $46 \%$, but only $5 \%$ of these were directly related to the anidulafungin (Krause et al 2004a). The most common adverse events included hypotension (13\%), vomiting (13\%), constipation (11\%), nausea (11\%) and pyrexia (11\%), but none are dose dependent. In over 1700 doses of anidulafungin given, no systemic infusion-related adverse reactions or anaphylactic reactions occurred. In another study, treatment related adverse events were reported in only $9.3 \%$ of patients (Krause et al 2004b). One patient reported subjective "flushing" after the infusion, although no other histamine-related reactions have been documented. Minor elevations in hepatic and hematological parameters were also seen. However, in the recently completed invasive candidiasis trial, 15 of 127 patients receiving anidulafungin reported an adverse event resulting in discontinuation of the drug (Reboli et al 2007). Common adverse events related to the use of anidulafungin are summarized in Table 3. 
Table 3 Adverse events (AE) associated with anidulafungin use in clinical trials

\begin{tabular}{|c|c|c|}
\hline & $\begin{array}{l}\text { Anidulafungin } 50 \text { mg } \\
\text { (Esophagitis Study) } \\
\mathrm{N}=300(\%)\end{array}$ & $\begin{array}{l}\text { Anidulafungin } 100 \mathrm{mg} \\
\text { (Candidemia Study) } \\
\mathrm{N}=131(\%)\end{array}$ \\
\hline Patients with $\geq I$ treatment-related $A E$ & $43(14.3)$ & $32(24.4)$ \\
\hline \multicolumn{3}{|l|}{ Gastrointestinal system } \\
\hline Nausea & $3(1.0)$ & NR \\
\hline Diarrhea & NR & $4(3.1)$ \\
\hline \multicolumn{3}{|l|}{ Blood and lymphatic system } \\
\hline Neutropenia & $3(1.0)$ & $N R^{a}$ \\
\hline Leukopenia & $2(0.7)$ & NR \\
\hline \multicolumn{3}{|l|}{ Investigations } \\
\hline Elevated $\gamma$-glutamyl transferase & $4(1.3)$ & NR \\
\hline Elevated alanine aminotransferase & 0 & $3(2.3)$ \\
\hline Elevated aspartate aminotransferase & $\mathrm{I}(0.3)$ & $\mathrm{I}(0.8)$ \\
\hline Elevated alkaline phosphatase & NR & $2(1.5)$ \\
\hline Elevated hepatic enzymes & NR & $2(1.5)$ \\
\hline \multicolumn{3}{|l|}{ Nervous system } \\
\hline Headache & $4(1.3)$ & NR \\
\hline \multicolumn{3}{|l|}{ Skin and subcutaneous tissue } \\
\hline Rash & $3(1.0)$ & NR \\
\hline
\end{tabular}

aNR: Not reported in study population.

Based on studies reported in Krause 2004a, 2004b.

\section{Anidulafungin drug interactions}

As discussed above, anidulafungin elimination occurs through non-enzymatic reactions and degradation through bile. Anidulafungin is not an inducer, inhibitor, or substrate, of the cytochrome P450 enzyme system. Therefore, little or no drug interactions are expected. It is not surprising, then, that when anidulafungin was co-administered with voriconazole to 18 healthy male volunteers, neither drug affected the other's pharmacokinetics (Dowell et al 2005). In another study, cyclosporine resulted in a $22 \%$ increase in anidulafungin drug exposure after 4 days of therapy. However, this increase was judged not to be clinically significant. Finally, no drug interactions have been found either with rifampin or amphotericin B (Dowell et al 2004), but no published data are present for interaction with other agents such as antiretroviral drugs that would be expected to be co-administered in patients with AIDS, as well as for other common problem agents such as phenytoin and calcium channel blockers.

\section{Anidulafungin's place in therapy}

There are several lines of evidence to support anidulafungin's role as primary antifungal therapy for candidiasis. First, in vitro studies have demonstrated broad susceptibility to anidulafungin among Candida species isolated from patients. Second, anidulafungin is fungicidal against Candida. This makes anidulafungin and other echinocandins, at least in theory, superior to fungistatic drugs such as fluconazole. Third, in vivo PK-PD studies have demonstrated superior efficacy, defined as maximal microbial kill, when compared to fluconazole, regardless of the fluconazole susceptibility of the Candida species. Fourth, there are adequate randomized controlled trials that have been performed to demonstrate anidulafungin efficacy in patients.

The efficacy of anidulafungin in the therapy of esophageal candidiasis has been compared to fluconazole as discussed earlier. However, fluconazole is still clearly the first choice. Despite fluconazole's theoretical limitations, it performed equivalent to anidulafungin at the end of therapy time point, and may even be superior when it comes to relapse rates. Given that the largest number of patients in whom esophageal candidiasis occurs is of AIDS patients who live in those countries of the globe that can least afford expensive therapy, fluconazole is a better choice even if equal efficacy to anidulafungin were to be postulated. At the current moment, a possible role would be to use anidulafungin as alternative therapy to fluconazole in those cases of poor tolerance to fluconazole or esophageal candidiasis due to fluconazole-resistant $\mathrm{Can}$ dida. However, given anidulafungin's safety, its predictable pharmacokinetics, few drug - drug interactions, and primary efficacy, it should probably be used ahead of amphotericin $\mathrm{B}$ as alternative therapy. The goals of therapy with anidulafungin, as for the fluconazole, would be total resolution of 
symptoms. We would like to point out that in patients with AIDS-associated esophagitis ART is indispensable.

Anidulafungin should be considered as part of first-line or primary therapy for candidemia and invasive candidiasis in non-neutropenic patients. This is a role it occupies with fluconazole and amphotericin B, as well as caspofungin and micafungin. The first important question is whether the anidulafungin should be considered the first choice, ahead of fluconazole and amphotericin B preparations. This is a controversial topic, as recently pointed out by Sobel and Revankar (Sobel and Revankar 2007). Different experts will reach different conclusions. We would like to argue that based on safety, anidulafungin should be considered preferable to amphotericin-B based compounds for the treatment of candidemia and invasive candidiasis. The question then becomes whether anidulafungin should be used ahead of fluconazole. Several facts should be considered. First, a randomized controlled study did show superiority of anidulafungin to fluconazole in the "gold standard" intention-totreat analysis. Second, the rates of persistent candidemia at the end of therapy was $6.3 \%$ with anidulafungin versus $14.4 \%$ with fluconazole $(\mathrm{p}=0.06)$. Third, the average global response rates to fluconazole in several clinical studies of candidemia have been persistently around 50\%-60\%, except in the study by Rex et al in 1994, while those of $100 \mathrm{mg}$ of anidulafungin and other echinocandins are often around 70\%-80\%, or even higher (Rex et al 1994; Phillips et al 1997; Rex et al 2003; Krause et al 2004a; Kuse et al 2007; Reboli et al 2007; Pappas et al 2007). Fourth, in vivo PK-PD studies have demonstrated that anidulafungin is actually more efficacious than fluconazole. On the other hand, the center effect in the anidulafungin randomized study cannot be discounted, as discussed above. In addition, efficacy against $C$. krusei was not studied. Furthermore, the significant differences in efficacy between anidulafungin and fluconazole were seen mainly in patients with $C$. albicans and C. tropicalis, with success rates of $81 \%$ and $93 \%$ in the anidulafungin arm versus $62 \%$ and $50 \%$ in the fluconazole arm. Superiority in patients treated with other Candida species therefore needs further investigation. However, there is an ethical dimension to be taken into consideration if anidulafungin is a potentially superior therapy. Ethical principles would seem to point to use of anidulafungin instead of fluconazole, given similar safety profiles with fluconazole and therapy shown to be superior in a randomized study. Of course, in those situations where the patients cannot afford anidulafungin, or it is for some reason not available, fluconazole should be the first line of therapy. This brings us to a final and rather fascinating question, which is whether other echinocandins can simply be substituted for anidulafungin. Caspofungin was compared to amphotericin B deoxycholate in a randomized controlled trial of patients with invasive candidiasis, and at the end of intravenous therapy demonstrated equivalency in the modified intention to treat analysis, and superiority (81\% versus $65 \%$ response) if only patients who met criteria for evaluation were analyzed (Mora-Duarte et al 2002). Recently, another randomized controlled trial compared micafungin to liposomal amphotericin B in patients with invasive candidiasis, and demonstrated equivalence (90\% versus $90 \%$ response) (Kuse et al 2007). Therefore, all three echinocandins have been demonstrated to have excellent efficacy in the treatment of invasive candidiasis, and as of yet all three should be considered first line therapy for invasive candidiasis.

In patients with candidemia and invasive candidiasis and neutropenia, there is insufficient data to recommend anidulafungin. In the dose ranging study by Krause et al for example, there were only 16 of 120 (13\%) patients with neutropenia, while in the study of anidulafungin versus fluconazole, there were only 7 of 245 patients with neutropenia (Krause el al 2007). Therefore, there is insufficient clinical data as of yet to recommend anidulafungin for candidemia in patients with neutropenia. In these patients, micafungin, caspofungin and lipid preparations of amphotericin B should be used (MoraDuarte et al 2002; Kuse et al 2007).

There have been very few reports, if any, of the use of anidulafungin in the treatment of Candida endocarditis, chronic disseminated candidiasis, osteomyelitis, urinary candidiasis or meningitis. The penetration of the drug into bone and many such sites is still poorly defined, but central nervous system penetration is known to be poor. It is also expected that there would be low concentrations achieved in urine, so that anidulafungin is not expected to work in Candida urinary tract infections. However, it is intriguing that three cases have been published in which another echinocandin, caspofungin, was efficacious in the treatment of complicated and ascending $C$. glabrata infection, even when that echinocandin also does not, in theory, achieve high concentrations in urine (Sobel et al 2007). However, prospective clinical studies with anidulafungin or any other echinocandin for that matter are lacking. Thus, at the present moment anidulafungin is not yet recommended for therapy at any of these sites.

Data are also lacking on the use of anidulafungin therapy for invasive aspergillosis. An open label, non-comparative study of the safety and efficacy of anidulafungin plus liposomal amphotericin B for the treatment of aspergillosis has 
been completed, but results have not yet been published in full. Details of this trial and the partial results can be found at Clinical Trials.gov (NCT00037206). Thus, the clinical utility of anidulafungin for invasive aspergillosis remains undefined.

\section{Conclusion}

Anidulafungin is a new echinocandin that has been introduced for use in patients with Candida and Aspergillus infections. Susceptibility data, in vivo PK-PD studies, and clinical studies support a role in the treatment of esophageal candidiasis in those patients who can not tolerate azole therapy or have azole-resistant Candida, and a role as first-line therapy for treatment of candidemia and invasive blood stream infection. However, data are lacking for use in other clinical scenarios, and in treatment of invasive aspergillosis.

\section{References}

Alexander BD, Schell WA, Miller JL, et al. 2005. Candida glabrata fungemia in transplant patients receiving voriconazole after fluconazole. Transplantation, 80:868-71.

Andes D, Marchillo K, Lowther J, et al. 2003. In vivo pharmacodynamics of HMR 3270, a glucan synthase inhibitor, in a murine candidiasis model. Antimicrob Agents Chemother, 47:1187-92.

Arathoon EG, Gotuzzo E, Noriega LM, et al. 2002. Randomized, doubleblind, multicenter study of caspofungin versus amphotericin B for treatment of oropharyngeal and esophageal candidiases. Antimicrob Agents Chemother, 46:451-7.

Buell D, Kovanda L, Drake T, et al. 2005. Alternative day dosing of micafungin in the treatment of esophageal candidiasis. 45th Interscience Conference on Antimicrobial Agents and Chemotherapy, Abstract M-719.

Clancy CJ, Barchiesi F, Falconi DiFrancesco L, et al. 2000. Clinical manifestations and molecular epidemiology of late recurrent candidemia, and implications for management. Eur J Clin Microbiol Infect Dis, 19:585-92.

de Wet N, Llanos-Cuentas A, Suleiman J, et al. 2004. A randomized, doubleblind, parallel-group, dose-response study of micafungin compared with fluconazole for the treatment of esophageal candidiasis in HIV-positive patients. Clin Infect Dis, 39:842-9.

Dowell J, Stogniew M, Krause D. 2003. Anidulafungin dosage adjustments are not required for patients with hepatic and/or renal impairment. 13th European Congress of Clinical Microbiology and Infectious Diseases.

Dowell JA, Knebel W, Ludden T, et al. 2004. Population pharmacokinetic analysis of anidulafungin, an echinocandin antifungal. J Clin Pharmacol, 44:590-8.

Dowell JA, Schranz J, Baruch A, et al. 2005. Safety and pharmacokinetics of coadministered voriconazole and anidulafungin. J Clin Pharmacol, 45:1373-82.

Dowell JA, Stogniew M, Krause D, et al. 2003. Anidulafungin (ANID) pharmacokinetic (PK)/Pharmacodynamic (PD) correlation: Treatment of esophageal candidiasis. 43rd Interscience Conference of Antimicrobial Agents and Chemotherapy, Abstract A-1578.

Espinel-Ingroff A. 1998. Comparison of In vitro activities of the new triazole SCH56592 and the echinocandins MK-0991 (L-743, 872) and LY303366 against opportunistic filamentous and dimorphic fungi and yeasts. J Clin Microbiol, 36:2950-6.

Espinel-Ingroff A. 2003. In vitro antifungal activities of anidulafungin and micafungin, licensed agents and the investigational triazole posaconazole as determined by NCCLS methods for 12,052 fungal isolates: review of the literature. Rev Iberoam Micol, 20:121-36.
Groll AH, Mickiene D, Petraitiene R, et al. 2001. Pharmacokinetic and pharmacodynamic modeling of anidulafungin (LY303366): reappraisal of its efficacy in neutropenic animal models of opportunistic mycoses using optimal plasma sampling. Antimicrob Agents Chemother, 45:2845-55.

Gumbo T, Ikeda F, Louie A. 2007a. Glucan synthase inhibitors. In: Nightingale CH, Murakawa T, Ambrose PG, Drusano GL, eds. Antimicrobial Pharmacodynamics in Theory and Clinical Practice. 2nd ed. New York: Informa Healthcare. p. 355-77.

Gumbo T, Chemaly RF, Isada CM, et al. 2002. Late complications of Candida (Torulopsis) glabrata fungemia: description of a phenomenon. Scand J Infect Dis, 34:817-8.

Gumbo T, Drusano GL, Liu W, et al. 2006. Anidulafungin pharmacokinetics and microbial response in neutropenic mice with disseminated candidiasis. Antimicrob Agents Chemother, 50:3695-700.

Gumbo T, Isada CM, Hall G, et al. 1999. Candida glabrata Fungemia. Clinical features of 139 patients. Medicine (Baltimore), 78:220-7.

Gumbo T, Drusano GL, Liu W, et al. 2007b. Once-weekly micafungin therapy is as effective as daily therapy for disseminated candidiasis in mice with persistent neutropenia. Antimicrob Agents Chemother, 51:968-74.

Krause DS, Reinhardt J, Vazquez JA, et al. 2004a. Phase 2, randomized, dose-ranging study evaluating the safety and efficacy of anidulafungin in invasive candidiasis and candidemia. Antimicrob Agents Chemother, 48:2021-4.

Krause DS, Simjee AE, van Rensburg C, et al. 2004b. A randomized, double-blind trial of anidulafungin versus fluconazole for the treatment of esophageal candidiasis. Clin Infect Dis, 39:770-75.

Kurtz MB, Abruzzo G, Flattery A, et al. 1996. Characterization of echinocandin-resistant mutants of Candida albicans: genetic, biochemical, and virulence studies. Infect Immun, 64:3244-51.

Kuse ER, Chetchotisakd P, da Cunha CA, et al. 2007. Micafungin versus liposomal amphotericin B for candidaemia and invasive candidosis: a phase III randomised double-blind trial. Lancet, 369:1519-27.

Louie A, Deziel M, Liu W, et al. 2005. Pharmacodynamics of caspofungin in a murine model of systemic candidiasis: importance of persistence of caspofungin in tissues to understanding drug activity. Antimicrob Agents Chemother, 49:5058-68.

Mora-Duarte J, Betts R, Rotstein C, et al. 2002. Comparison of caspofungin and amphotericin B for invasive candidiasis. N Engl J Med, 347:2020-9.

Nasser RM, Melgar GR, Longworth DL, et al. 1997. Incidence and risk of developing fungal prosthetic valve endocarditis after nosocomial candidemia. Am J Med, 103:25-32.

Nguyen MH, Peacock JE Jr, Morris AJ, et al. 1996. The changing face of candidemia: emergence of non-Candida albicans species and antifungal resistance. Am J Med, 100:617-23.

Oakley KL, Moore CB, Denning DW. 1998. In vitro activity of the echinocandin antifungal agent LY303, 366 in Comparison with itraconazole and amphotericin B against Aspergillus spp. Antimicrob Agents Chemother, 42:2726-30.

Pappas PG, Rotstein CM, Betts RF, et al. 2007. Micafungin versus caspofungin for treatment of candidemia and other forms of invasive candidiasis. Clin Infect Dis, 45:883-93.

Pfizer Inc. 2006. Final package insert for ERAXIS (anidulafungin) for injection. New York.

Phillips P, Shafran S, Garber G, et al. 1997. Multicenter randomized trial of fluconazole versus amphotericin B for treatment of candidemia in non-neutropenic patients. Canadian Candidemia Study Group. Eur J Clin Microbiol Infect Dis, 16:337-45.

Reboli AC, Rotstein C, Pappas PG, et al. 2007. Anidulafungin versus fluconazole for invasive candidiasis. $N$ Engl J Med, 356:2472-82.

Rex JH, Bennett JE, Sugar AM, et al. 1994. A randomized trial comparing fluconazole with amphotericin B for the treatment of candidemia in patients without neutropenia. Candidemia Study Group and the National Institute. N Engl J Med, 331:1325-30.

Rex JH, Pappas PG, Karchmer AW, et al. 2003. A randomized and blinded multicenter trial of high-dose fluconazole plus placebo versus fluconazole plus amphotericin B as therapy for candidemia and its consequences in nonneutropenic subjects. Clin Infect Dis, 36:1221-8. 
Serrano MC, Valverde-Conde A, Chavez MM, et al. 2003. In vitro activity of voriconazole, itraconazole, caspofungin, anidulafungin (VER002, LY303366) and amphotericin B against Aspergillus spp. Diagn Microbiol Infect Dis, 45:131-5.

Sobel JD, Bradshaw SK, Lipka CJ, et al. 2007. Caspofungin in the treatment of symptomatic candiduria. Clin Infect Dis, 44:e46-e49.

Sobel JD, Revankar SG. 2007. Echinocandins - first-choice or first-line therapy for invasive candidiasis? $N$ Engl J Med, 356:2525-6.

Trissel LA, Ogundele AB. 2005. Compatibility of anidulafungin with other drugs during simulated Y-site administration. Am J Health Syst Pharm, 62:834-7.

Wiederhold NP, Kontoyiannis DP, Chi J, et al. 2004. Pharmacodynamics of caspofungin in a murine model of invasive pulmonary aspergillosis: evidence of concentration-dependent activity. $J$ Infect Dis, 190:1464-71. 